\title{
Fairness Comparison of TCP Variants over Proactive and Reactive Routing Protocol in MANET
}

\author{
Nahida Nigar1, Muhammad Anwarul Azim² \\ ${ }^{1}$ Department of Computer Science and IT, Southern University Bangladesh, Bangladesh \\ ${ }^{2}$ Department of Computer Science and Engineering, University of Chittagong, Bangladesh
}

\section{Article Info \\ Article history: \\ Received Jul 25, 2017 \\ Revised Jan 3, 2018 \\ Accepted Apr 4, 2018}

Keyword:

Congestion control

MANET

Routing protocol

TCP variants

\begin{abstract}
Mobile ad hoc networks (MANETs) are applicable in an infrastructureless environment where the mobile devices act as routers and intermediate nodes are used to transfer segments to their final destination. As Transmission control protocol (TCP) was originated for Internet with fundamentally different properties, faces serious challenges when used in mobile ad hoc networks. TCP functionality degrades, due to special properties of MANET such as route failure because of significant change of network topology and link errors. TCP uses Congestion Control Algorithms; TCP Vegas is one of them which claim to have better throughput comparing with other TCP variants in a wired network. Fairness issues of TCP Variants in MANET including existing routing protocol are still unsolved. To determine the best TCP Variants in MANET environment over renowned routing protocol is the main objective of this paper. A Study on the throughput fairness of TCP Variants namely, Vegas, Reno, New Reno, SACK, FACK, and Cubic are performed via simulation experiment using network simulator (ns-2) over existing routing protocol, named, AODV, AOMDV, DSDV, and DSR. This fairness evaluation of TCP flows arranged a contrast medium for the TCP Variants using stated routing protocol in MANET. However, TCP Vegas obtain unfair throughput in MANET. The simulation results show that TCP Reno outperforms other TCP variants under DSDV routing protocol.
\end{abstract}

Copyright $@ 2018$ Institute of Advanced Engineering and Science. All rights reserved.

\section{Corresponding Author:}

Nahida Nigar,

Department of Computer Science and Engineering,

Southern University Bangladesh,

Mehedibag, Chittagong, Bangladesh.

Email: n.nigar.87@gmail.com

\section{INTRODUCTION}

Mobile ad hoc network (MANET) [1] nodes are furnished with wireless transmitters and receivers using highly directional or Omni-directional antennas, probably steerable, or some combination thereof. They are dynamic in nature, autonomous and purpose-specific. In MANET there is no master-slave relationship that exists which compares greatly with fixed wireless networks. Each node acts as a router, requires routing algorithms such as Destination Sequenced Distance Vector (DSDV) [2], Ad-hoc On-demand Distance Vector (AODV) [3], Ad-hoc On-demand Multipath Distance Vector Routing (AOMDV) [4] protocol and Dynamic Source Routing (DSR) [5] to discover and maintain routes to forwards packets to other nodes. Thus, MANETs are multi-hop wireless networks by nature.

The protocols to be used in MANET may differ, depending upon the capabilities of the devices, packet drop rate and other factors. Transmission Control Protocol provides a reliable packet delivery over an unreliable network as it is a connection-oriented protocol. Originally TCP provides sender-side flow control mechanism by using the maximum allowed window size, advertised by the receiver and the sender is only allowed to send new packets after receiving acknowledgment for the previous packet. Many of TCP variants 
have been proposed [6]. Among these TCP variants, TCP Vegas claims to have a better throughput [7] in both wired [8] and wireless network [9], [10]. Theoretically, TCP should not depend on the technology of the underlying infrastructure. In particular, TCP is independent of the Internet Protocol (IP). In practice, most TCP deployments are specific to wired networks. Usually, congestion on Internet concentrated on one single router, whereas congestion in MANET's affects a whole area because of the shared medium. Ignoring the properties of wireless transmission can lead to TCP implementation with poor performance.

Despite the fact that considerable simulation work has been done, still more investigation is needed in the fairness of the TCP traffic and mobility models. This paper present succinct view of the prior reason for six TCP Variants interpretation over four routing protocol in MANET, and cover the state of the art in the solution spectrum.

This paper simulates a wireless network where the nodes move randomly with TCP flows on simulation topology. Difficulty exists to analyze fairness of TCP flows using TCP variants over routing protocols based only on mathematical and theoretical calculations. There are a lot of different parameters that need to be considered. Implementation of wireless network topology using simulators helps to overcome these difficulties. The simulation of the MANET gives a better perspective which helps us to reveal parameters influence on the MANET's behavior.

Using advanced mathematical software tools as GNUPLOT, simulations results can be easily analyzed. After receiving all the results of simulations conclusions done. These conclusions will help in future to design more complex and large-scale mobile ad hoc networks.

Research is still going on MANETs which involve efficient routing considering the fact that the topology changes so frequently over time. Fairness issues of TCP Variants in MANET including existing routing protocol are still unsolved.

Internet protocol involves routing protocols that are categorized into pro-active, reactive and hybrid routing protocols [11], and most appropriate routing protocol can be identified by measuring different factors, namely: a) quality of service, b) scalability, and c) traffic and mobility models. Analytical studies have been done on various different routing protocols that involve TCP, CBR and VBR (Variable Bit Rate) traffic models [12], [13]. In wired network packet losses indicate congestion in a link, but analysis has revealed that in the wireless network, performance of TCP traffic models not only depend on packet losses but also link failure and late acknowledgment play a significant role to indicate collision in wireless scenario due to the frequent topology changes. Architecturally, TCP is implemented for static node networks. Clarification of best routing protocol in the response to segment loss and link failure is also unknown, still main causes of packet loss in MANET cannot be found. An extension to the AODV protocol is Ad-hoc On-demand Multipath Distance Vector Routing (AOMDV) [4] protocol is introduced for computing multiple loop-free and link disjoint paths [14].

In Ad-hoc network, congestion does not always occur for packet loss. But several types of losses occur in a wireless network not only related to congestion [15], but also for it dynamic nature. To improve TCP performance over ad hoc network, a lot of development and enhancement have been proposed.

The previous studies [7], [9], [10] show that TCP Vegas [8] effectuate better throughput than other TCP variants both in wired and mobile ad hoc network. However, this is only true in the wired network that fully involves TCP Vegas. But in our analysis, the authors investigate the effects of mobile ad hoc network among six Variants including TCP Vegas over four routing protocol where the performance of TCP Vegas diminishes drastically. TCP Vegas failed to attain fair bandwidth allotment when competing with other TCP sources. In this paper, the fairness issues of different TCP variant like TCP Reno, TCP New Reno, TCP SACK, TCP Vegas, TCP FACK and TCP Cubic are analyzed when running over different routing protocol like DSDV, AODV, AOMDV and DSR using ns-2 [16]. In our analysis, among TCP variants TCP Reno achieves better throughput comparing with other variants under DSDV routing protocol in all aspects.

Routing protocol classification in MANET's can be done depending on routing scheme and network infrastructure [17], [18]. Internet distance-vector and link-state protocols are used to implement proactive routing protocols, also known as traditional routing protocols [2], [19], [20] preserve routes to all nodes. They react to any change in the topology and maintain consistent and updated routing table to each node in the network. Destination Sequenced Distance Vector (DSDV) [2] is a representative among popular proactive protocols. To reduce the overhead, alternative reactive routing protocols [3], [21] is used to determine the route in IP. The topology size, the capacity of the link, connectivity patterns and portability affects the routing protocols behavior. Among on-demand routing protocols, AODV [3] and DSR [22] are the most prominent reactive protocol and many variations of AODV are being enhanced. AOMDV [4] extends the prominence of AODV to discover multi-path among a sender and receiver.

The interaction of MANET with the TCP protocol structure may lead to impulsive phenomena like severe unfairness problem between simultaneous TCP flows. The following TCP variants: Reno [21], New Reno [23], Vegas [8], SACK [24], FACK [25] and CUBIC [26] perform accordingly based on their core 
mechanisms. The DSDV protocol [2] is a protocol with extensions maintain a routing table which records the shortest path for each node. A sequence number is maintained for the destination is used to avert routing loops.

AODV, a reactive improvement of DSDV, lessen routing overhead by creating routes on demand [3]. The route request that initiated on demand is forwarded by the nodes until a fresh route location remains discovered. A restraint of AODV is it only works with symmetric links and may be challenging to satisfy in MANETs. AOMDV [4] enhance the eminence of AODV, represents a timer-based protocol where mobile nodes can respond to topology changes and link breaks.

DSR [5] is a loop-free, source based on-demand routing protocol requires larger memory requirements along with potentially larger control overhead than AODV. Unlike AODV, DSR packet must carry full routing path information. DSR can utilize both symmetric and asymmetric link where nodes maintain multiple routes in their cache to the destination which is crucial as link failure.

TCP Variants [8], [21], [23]-[26] intertwined with four common TCP congestion control deployment named Slow start, Congestion Avoidance, Fast Retransmit and Fast Recovery. Slow Start mechanism increases the congestion window (cwnd) size by one for each acknowledgment. Congestion avoidance phase rebate the sender's window size by half at the observation of loss and increase the cwnd at the rate of about one packet per round trip time (RTT). Fast retransmit phase is introduced to recover packet loss, do not wait for retransmit timer go off, and retransmit the packet if a triple duplicate acknowledgment is achieved. In the Fast recovery phase, after prevailing duplicate acknowledgment, one packet may be left, it'll switch to congestion avoidance phase, but do not jump down to slow start.

TCP Reno [21] implements all the basic algorithm of TCP congestion control. However, it is an extended version of TCP. It uses duplicate acknowledgment and triple duplicate acknowledgment to identify a packet loss. A modest improvement of TCP Reno is TCP New RENO [23]. Unlike Reno, it detects multiple packet losses and doesn't exit fast-recovery until all the outstanding data in fast recovery phase is acknowledged, proceeds after receiving a fresh ACK as in Reno.

TCP Vegas [8] is a congestion avoidance algorithm achieves $40 \%-70 \%$ better throughput than Reno only if routers buffer is not heavily utilized. Comparing with Reno, it is less aggressive and path rerouting changes the baseRTT, causes throughput loss. The basic problem in TCP deployment is that cumulative acknowledgment provides less information. Selective Acknowledgment [24] adds a bitmask of packet arrived which is implemented as a TCP option, negotiated during handshake. Though the acknowledged sequence number is still cumulative.

Forward acknowledgment (FACK) is designed based on TCP SACK option without incurring a transmission timeout [25]. TCP CUBIC [26] maintain two states, named the Steady state, where window grows faster for a window reduction to maximum window size, it slows down its growth and a Probing state, where window grows slowly around maximum window size enhances the stability. In [27] the researcher has discussed about the open issues of techniques to mitigate the security problem using Swarm Intelligence. In [28] the researcher has introduced a novel topological based approach which is compatible with largescale MANET and shows better outcome with respect to AODV and DSDV. A performance study of Intra and Inter-group MANET routing protocol [29] shows that there is no significant impact of varying of nodes on standard performance evaluation matric.

\section{MANET SIMULATION WITH NS-2}

Mobile Ad hoc Networks (MANETs) environment is simulated by using network simulator [16] 2.35 version for this experiment. The simulation has been developed to emphasize the impact of TCP flows using different TCP variants over different routing protocol where FTP connections are used as TCP flows. The simulation environment as shown in Figure 1 formed a MANET over a 1000 x1000 meters grid consist of 30 nodes distributed randomly. In this topology, for constant movement of nodes throughout the simulation we set the pause time to 0. All mobile nodes in the network are configured to run AODV, AOMDV, DSDV and DSR protocols and multiple FTP sessions using six TCP variants namely TCP Vegas, TCP Reno, TCP New Reno, TCP SACK, TCP FACK and TCP Cubic. The simulation topology is shown in Figure 1. 


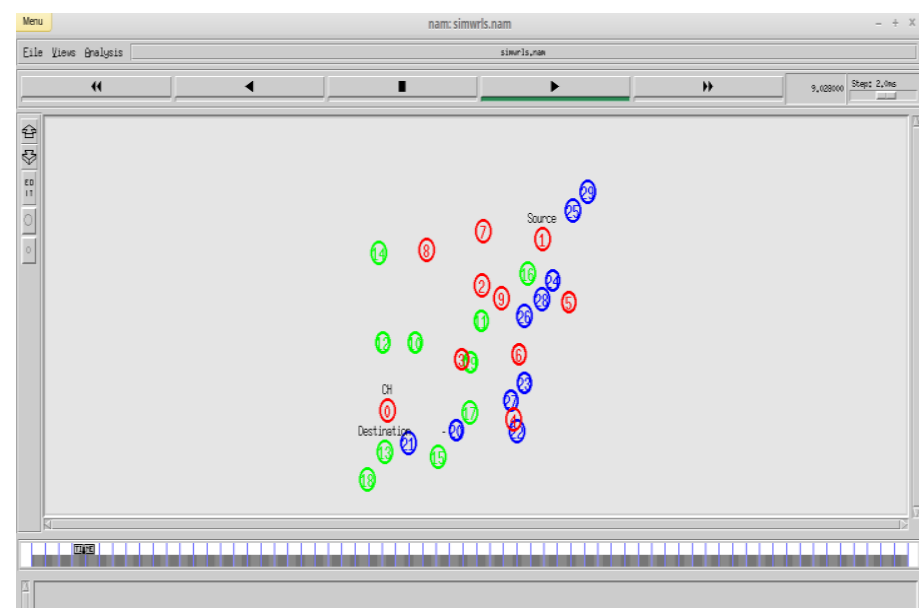

Figure 1. MANET Simulated Topology in NS2 Environment

The fairness of TCP Variants in wireless mobile network is evaluated in our simulation experiment, using six TCP variants on four routing protocol of which each node share FTP connections randomly. In each run throughput fairness and packet drop is calculated for each of the four routing protocols at 30 node densities for 150 seconds. As in MANET, each mobile node acts as both host and router there is no specific sender or receiver. Sender and receiver are chosen randomly by calculating shortest possible path. The parameters considered for this simulation experiment are shown in Table 1. To examine the throughput fairness of TCP flows and packet loss, the fairness comparison of TCP variants is evaluated over four routing protocol in MANET.

Table 1. Simulation Parameters

\begin{tabular}{cc}
\hline Parameter & Values \\
\hline Topology Area & 1000x1000 meter ${ }^{2}$ \\
Channel Type & Wireless Channel \\
Mac Type & IEEE 802.11 \\
Network Interface Type & Wireless Physical Layer \\
Ad hoc Routing Protocol & AODV, AOMDV, DSDV, DSR \\
Total Node & 30 \\
Movement Speed & $0-20 \mathrm{~m} / \mathrm{s}$ \\
Traffic Model & TCP, FTP \\
TCP Variants & TCP Reno, TCP New Reno, TCP Vegas, \\
Packet Size & TCP SACK, TCP FACK, TCP CUBIC \\
Node Placement & 1024 Byte \\
Pause Time & Random Waypoint \\
Antenna Type & 0 \\
Buffer Size & Omni Antenna \\
& 200 \\
\hline
\end{tabular}

\section{SIMULATION RESULT AND ANALYSIS}

In this section, we present the simulation results and the fairness comparison of TCP Variants is analyzed for mobile ad hoc network by using 30 node densities over four different routing protocols. Our analysis shows that among six TCP variants TCP Reno performed better in MANET for DSDV protocol. On the other hand, TCP Vegas achieves unfair throughput in mobile ad hoc network which is by design claimed to have better throughput in any network [7], [9], [10]. By measuring throughput fairness and packet drop behavior of TCP flows we can understand how TCP Variants reacts to the mobile ad hoc network conditions which will help us to understand how reactive and proactive routing protocol has facilitated TCP Variants operation.

\subsection{Throughput fairness of TCP flows}

The rate of successfully transmitted data per second in the network during simulation is known as 
throughput. Fairness index can be calculated by the following Equation [30]:

$$
f(x)=\frac{\left(\sum_{i=1}^{n} x_{i}\right)^{2}}{n \cdot \sum_{i=1}^{n}\left(x_{i}\right)^{2}} \text { such that } f(x) \in(0,1)
$$

As throughput is a positive value, the throughput fairness of a set of TCP flows will always lie between 0 and 1 . If the index indicates 1 , the throughput fairness is high and each receiver will receive equal throughput. From Table 2, we can see that for mobile ad hoc network, the average throughput fairness of six TCP Variants over reactive and proactive routing protocol is analyzed using 30 nodes, each of the nodes moves randomly and deliver FTP traffic to their shortest path distance receiver. For all the mentioned set up, TCP Vegas obtain lowest average throughput compared with other TCP Variants as shown in Table 2.

TCP Vegas received the most unfair throughput over four routing protocol. In our simulation, eight senders try to send packet at a time, as a consequence collisions occur so frequently which indicate congestion in mobile ad hoc network. As Reno flows are more aggressive, as a result, can achieve higher throughput comparing with other TCP Variant. A Simulation that includes Reno flow operating with reactive and proactive routing protocol has consistently better performance excluding with AOMDV protocol. In AOMDV protocol, TCP FACK gives better throughput. As previous studies [9] shows that reactive routing protocol AODV performs better than DSDV protocol, our analysis shows that TCP Variants adjust well with DSDV protocol comparing with other routing protocol.

The results show that as shown in Table 2 among six TCP variants, TCP Reno outperforms other TCP variant assisted with DSDV protocol. From Table 2, we can also see that Vegas cause's unfair distribution of throughput due to path changes so frequently results in the change of baseRTT. Although TCP Vegas privileges to have better throughput than other TCP Variants on MANET [10], [30], [31] with AODV [9], [32] than DSDV and DSR routing protocol. Our studies show that all TCP variants gives better throughput with DSDV routing protocol as shown in Table 2. Than TCP variants work well with DSR, then it is for AODV and at last for AOMDV. After TCP Reno, TCP CUBIC gives higher throughput over DSDV. After that, TCP FACK works well with AOMDV protocol. TCP Vegas gives most unfair throughput in MANET for both reactive and proactive routing protocol including AOMDV. Table 2 shows the average throughput (kbps) achieved by TCP flows in MANET.

Table 2. Average Throughput (kbps)

\begin{tabular}{ccccccc}
\hline Routing Protocol & Vegas & Reno & New Reno & SACK & FACK & Cubic \\
\hline AODV & 176.13 & 354.71 & 350.58 & 319.17 & 329.02 & 351.27 \\
AOMDV & 165.01 & 332.1 & 328.4 & 324.04 & 371.57 & 343.47 \\
DSDV & 178.37 & 446.8 & 392.92 & 369.68 & 386.79 & 404.25 \\
DSR & 186.41 & 373.6 & 361.53 & 334.87 & 366.11 & 368.72 \\
\hline
\end{tabular}

From the graph below as shown in Figure 2, it can be seen that throughput is more consistent for TCP Reno on DSDV protocol rather than TCP Vegas, Reno, NewReno, SACK, FACK, and CUBIC. After TCP Reno, it is good for TCP CUBIC and then for TCP New Reno and TCP FACK for AOMDV.

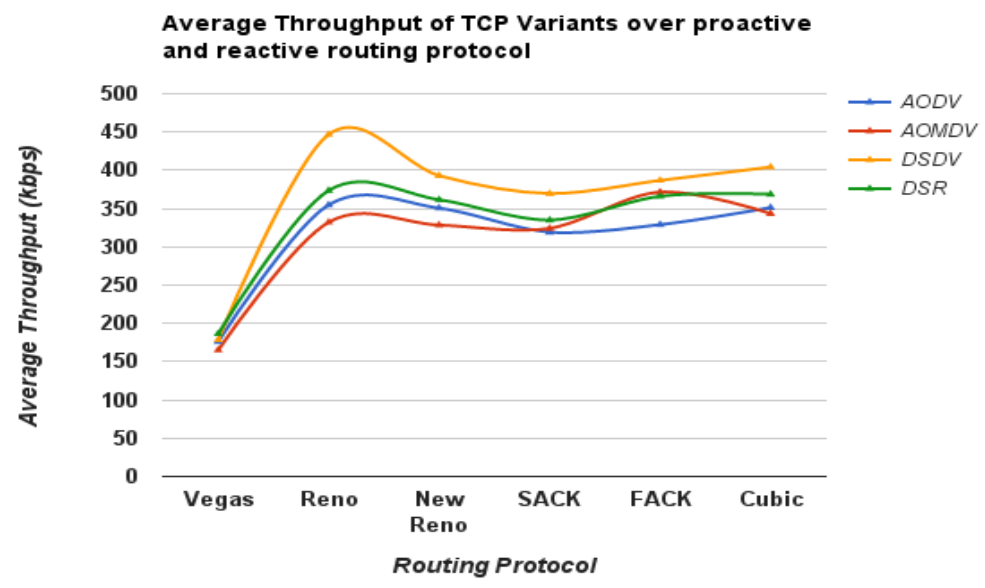

Figure 2. Average throughput (kbps) of TCP Variants over reactive and proactive routing protocol

Fairness Comparison of TCP Variants over Proactive and Reactive Routing Protocol ... (Nahida Nigar) 


\subsection{Packet drop rate}

TCP Vegas is a pro-active algorithm [7], [8] that reduce the congestion window when it experiences a packet loss and reduces throughput where for other variants it needs to loss multiple packets to find the available resource of the connection in wired topology. But in MANET as in implementation details, TCP Vegas suffers from less packet drop as shown in Table 3 but achieve unfair throughput compared with other TCP variants. TCP Vegas use packet loss as congestion indication, it reduces the congestion window immediately, as a consequence it remains less packet drop but cannot achieve high throughput in MANET for random movement of nodes.

Table 3. Packet Drop

\begin{tabular}{ccccccc}
\hline Routing Protocol & Vegas & Reno & New Reno & SACK & FACK & Cubic \\
\hline AODV & 412 & 552 & 565 & 683 & 586 & 530 \\
AOMDV & 356 & 362 & 421 & 502 & 344 & 419 \\
DSDV & 126 & 178 & 219 & 206 & 199 & 170 \\
DSR & 102 & 210 & 216 & 195 & 163 & 186 \\
\hline
\end{tabular}

From Figure 3, we can see that all TCP variants under AODV protocol suffer from highest packet drop. But packet drop behavior under DSDV protocol is more consistent compared with other protocol. DSR also suffer from less packet drop but unlike DSDV, TCP variants cannot achieve the highest throughput. Table 3 shows the average packet drop of TCP variants under reactive and proactive routing protocol.

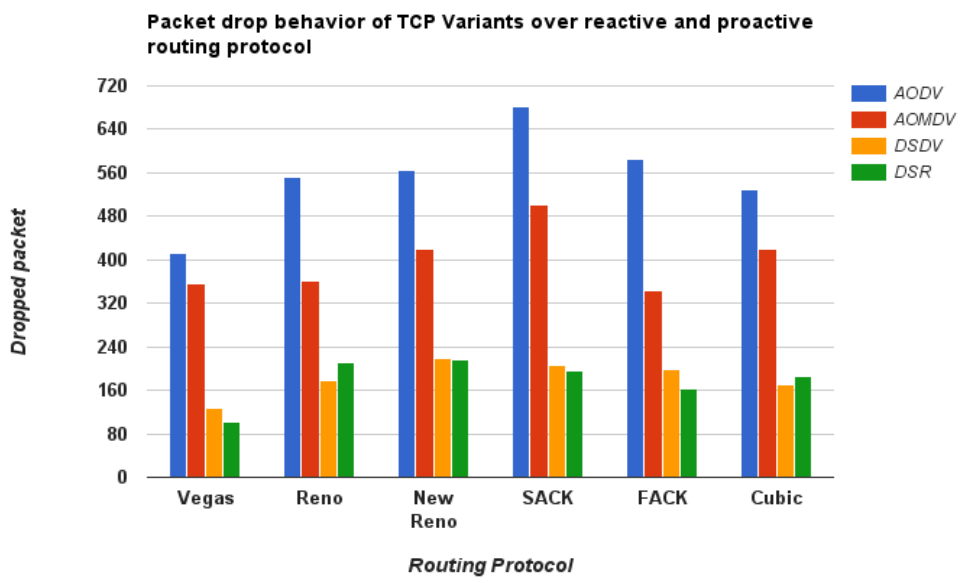

Figure 3. Packet Drop behavior of TCP Variants over reactive and proactive routing protocol

If data transfer from different nodes increases in simulation, the possibility of collisions increases, as a result, packet drop rate increases. Despite the best effort service of routing protocol, it is difficult to even distribution of mobile ad hoc network resources among flows that includes TCP traffic. Table 4 shows the average packet drop rate of the reactive and proactive routing protocol.

Table 4. Average Packet Drop Rate

\begin{tabular}{cc}
\hline Routing Protocol & Average Packet Drop Rate \\
\hline AODV & $41.4 \%$ \\
AOMDV & $35.7 \%$ \\
DSDV & $12.7 \%$ \\
DSR & $10.2 \%$ \\
\hline
\end{tabular}

\section{CONCLUSION}

The previous studies [7], [9], [10] show that TCP Vegas [8] effectuate better throughput than other TCP variants both in wired and mobile ad hoc network. However, this is only true in a wired network that fully involves TCP Vegas. But in our analysis, the authors investigate the effects of mobile ad hoc network 
using simulation experiments, where we evaluate the throughput fairness of TCP Variants over reactive and proactive routing protocol in mobile ad hoc network. From our analysis, we have found that TCP Vegas achieves unfair throughput comparing with other TCP variants over four of the routing protocol named AODV, AOMDV, DSDV, and DSR. It also showed an inconsistent performance for all four routing protocols as an average. From TCP variants, TCP Reno outperforms other TCP variants under DSDV routing protocol. However, TCP Cubic also observe better throughput than the other variants over the stated routing protocols. Though TCP Vegas gives better throughput in a wired network due to random mobility model, it takes the wrong measurement of baseRTT, whereas other stated TCP variants continue to increase their sender's window until multiple packet loss is detected. But in MANET the performance decreases inefficiently. As DSR responds quickly to link failure which circumvents TCP's deployment acknowledge at low pause time. The generic investigation from the simulation is that for throughput fairness of TCP flows and packet drop behavior existing DSDV, outperforms AODV, AOMDV, and DSR in more "stressful" topology like for an increased number of nodes and high mobility pattern. After DSDV, it is DSR that performs well with TCP variants. It is also confirmed that underlying routing protocol plays a significant role in throughput fairness of TCP variants in MANET.

\section{REFERENCES}

[1] Z J. Haas, et al., "Wireless ad hoc networks," Encyclopedia of Telecommunications, 2002.

[2] C. E. Perkins and P. Bhagwat, "Highly dynamic destination-sequenced distance-vector routing (DSDV) for mobile computers," ACM SIGCOMM computer communication review, vol. 24, no. 4, 1994.

[3] C. Perkins, et al., "Ad hoc on-demand distance vector (AODV) routing," No. RFC 3561, 2003.

[4] M. K. Marina and S. R. Das, "On-demand multipath distance vector routing in ad hoc networks," Network Protocols, 2001. Ninth International Conference on. IEEE, 2001.

[5] A. Ahuja, et al., "Performance of TCP over different routing protocols in mobile ad-hoc networks," Vehicular Technology Conference Proceedings, 2000. VTC 2000-Spring Tokyo. 2000 IEEE 51st, vol. 3, 2000.

[6] J. Postel, "Transmission Control Protocol Darpa Internet Program Protocol Specification," Request of Comment 793, Internet Engineering Task Force, 1981.

[7] L. S. Brakmo and L. L. Peterson, "TCP Vegas: End to end congestion avoidance on a global Internet," IEEE Journal on selected Areas in communications, vol. 13, no. 8, pp. 1465-1480, 1995.

[8] L. S. Brakmo, et al., "TCP Vegas: New techniques for congestion detection and avoidance," in SIGCOMM 94, London, UK, 1994.

[9] I. K. Tabash, et al., "Performance analysis of some TCP variants for routing over mobile ad hoc networks," International Journal of Advanced Computer Research, vol. 3, no. 3, pp. 52, 2013.

[10] M. M. Morshed, et al., "Performance comparison of TCP variants over AODV, DSDV, DSR, OLSR in NS-2," Informatics, Electronics \& Vision (ICIEV), 2012 International Conference on. IEEE, 2012.

[11] C. E. Perkins, “Ad hoc networking," Addison-Wesley Professional, 2008.

[12] S. Rajagopalan and C. C. Shen, "What does using TCP as an evaluation tool reveal about MANET routing protocols ?", Proceedings of the 2006 international conference on Wireless communications and mobile computing. ACM, 2006.

[13] D. Triantafyllidou and K. A. Agha, "Evaluation of TCP performance in MANETs using an optimized scalable simulation model," Modeling, Analysis, and Simulation of Computer and Telecommunication Systems, 2007. MASCOTS'07. 15th International Symposium on. IEEE, 2007.

[14] H. D. Trung, et al., "Performance evaluation and comparison of different ad hoc routing protocols," Computer Communications, vol. 30, no. 11, pp. 2478-2496, 2007.

[15] F. Ahmed, et al., "Performance Evaluation of TCP over Mobile Ad hoc Networks," arXiv preprint arXiv: 1002.2189, 2010.

[16] Ns-2 simulator, http://www.isi.edu/nsnam/ns.

[17] X. Hong, et al., "Scalable routing protocols for mobile ad hoc networks," IEEE network, vol. 16, no. 4, pp. 11-21, 2002.

[18] E. M. Royer and C. K. Toh, "A review of current routing protocols for ad hoc mobile wireless networks," IEEE personal communications, vol. 6, no. 2, pp. 46-55, 1999.

[19] C. C. Chiang, et al., "Routing in clustered multihop, mobile wireless networks with fading channel," Proceedings of IEEE SICON, vol. 97, 1997.

[20] S. Sung, et al., "Hierarchical clustering algorithm based on mobility in mobile ad hoc networks," International Conference on Computational Science and Its Applications, Springer Berlin Heidelberg, 2006.

[21] W. Stevens, et al., "RFC 2581: TCP Congestion Control," Consultant, 1999.

[22] D. Johnson, et al., "The dynamic source routing protocol (DSR) for mobile ad hoc networks for IPv4," No. RFC $4728,2007$.

[23] S. Floyd, et al., "The NewReno modification to TCP's fast recovery algorithm," 2004.

[24] M. Mathis, et al., "TCP selective acknowledgment options," No. RFC 2018, 1996.

[25] M. Mathis and J. Mahdavi, "Forward acknowledgement: Refining TCP congestion control," ACM SIGCOMM Computer Communication Review, vol. 26, no. 4, 1996. 
[26] S. Ha, et al., "CUBIC: a new TCP-friendly high-speed TCP variant," ACM SIGOPS Operating Systems Review, vol. 42, no. 5, pp. 64-74, 2008.

[27] P. K. Krishnappa and B. R. P. Babu, "Investigating Open Issues in Swarm Intelligence for Mitigating Security Threats in MANET," International Journal of Electrical and Computer Engineering, vol. 5, no. 5, 2015.

[28] R. Havinal, et al., "EASR: Graph-based Framework for Energy Efficient Smart Routing in MANET using Availability Zones," International Journal of Electrical and Computer Engineering, vol. 5, no. 6, 2015.

[29] D. S. Sisodia, et al., "A Performance Review of Intra and Inter-Group MANET Routing Protocols under Varying Speed of Nodes," International Journal of Electrical and Computer Engineering, vol. 7, no. 5, pp. 2721, 2017.

[30] M. Jehan, et al., "Vegas: Better performance than other TCP congestion control algorithms on Manets," International journal of computer networks (IJCN), vol. 3, no. 2, pp. 151-158, 2011.

[31] P. K. Meher and P. J. Kulkarni, "Analysis and Comparison of Performance of TCP-Vegas in MANET," Communication Systems and Network Technologies (CSNT), 2011 International Conference on. IEEE, 2011.

[32] N. Kaur, et al., "Simulation based Analysis of TCP Variants over MANET Routing Protocols using NS2," International Journal of Computer Applications (0975-8887), 2014.

\section{BIOGRAPHIES OF AUTHORS}

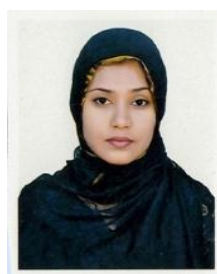

Nahida Nigar received her B.Sc.Eng. and M.Sc Engineering from University of Chittagong, Bangladesh in 2013 and 2016, respectively. She is now Lecturer in the Department of Computer Science and IT at Southern University Bangladesh, Chittagong, Bangladesh. Her research interests include Network Security, Wireless Communication, and Artificial Intelligence.

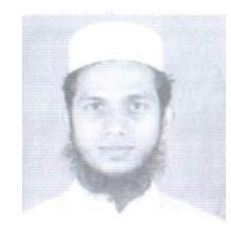

Muhammad Anwarul Azim is an Associate Professor of the Department of Computer Science and Engineering, University of Chittagong, Bangladesh. He received Master of Engineering degree from Department of Computer Engineering, Korea Aerospace University, Seoul, South Korea on February 2008 and Bachelor of Engineering degree from Department of Computer Science and Engineering, Shahjalal University of Science and Technology, Sylhet, Bangladesh. He has got a number of national and international conference and journal papers. His research interest includes but not limited to Security of Network, Ubiquitous Computing, Persistent Computing, Security Engineering, and Secure Software Engineering. 